\title{
Splenic transposition is superior to caudal shunt as a model of murine total hepatic ischemia
}

\author{
Tadashi Matsumoto, Philip A Efron, Hironori Tsujimoto, Sven K Tschoeke, \\ Ricardo Ungaro, Shiro Fujita, David P Foley, Alan Hemming and Lyle L Moldawer \\ Department of Surgery, University of Florida College of Medicine, Gainesville, FL, USA
}

\begin{abstract}
Murine total hepatic ischemia (THI) followed by reperfusion without shunting of the portal vein induces significant lethality in rodents due to intestinal congestion. Two methods have been promulgated to study THI and reperfusion in mice without intestinal congestion: subcutaneous splenic transposition which creates a portosystemic shunt via epigastric vessels, and a caudal shunt with $30 \%$ hepatectomy, which creates a portosystemic shunt via the small remnant of remaining caudal lobe. We compared outcome, inflammatory response and hepatic injury due to THI and reperfusion in these two models. Female C57BL/6 mice underwent ST, caudal shunt or no surgery prior to having $30 \mathrm{~min}$ of total hepatic ischemia followed by $60 \mathrm{~min}$ of reperfusion. Survival, surgical complications, serum AST/ALT and IL-6 were determined. Apoptotic and necrotic hepatocytes were identified by morphological criteria. Complication rates for the ST and caudal shunt procedures were 6.7 and $20 \%$, respectively. Subsequent mortality rates following THI and 60 min reperfusion were 5.9 and $50 \%$ in mice with ST and caudal shunt, respectively. Both groups had elevated serum AST/ALT concentrations. However, in mice undergoing caudal shunt, AST/ALT levels were also significantly increased even without THI. The number of apoptotic hepatocytes after THI and reperfusion in mice following caudal shunt was significantly higher compared with those of ST $(P<0.001)$. Both $\mathrm{ST}$ and caudal shunt can be used in models of THI and reperfusion to prevent significant lethality due to intestinal congestion. However, ST is a simple, safe and suitable model, whereas caudal shunt requires manipulation of the liver, and is associated with significant hepatic injury and morbidity.

Laboratory Investigation (2005) 85, 90-98, advance online publication, 22 November 2004; doi:10.1038/labinvest.3700210
\end{abstract}

Keywords: intestinal congestion; mouse; portal hypertension; splenic transposition; total hepatic ischemia and reperfusion

Total hepatic ischemia (THI) and reperfusion injury are inevitable consequences of various surgical maneuvers that occur during hepatobiliary surgery, liver transplantation and hepatic trauma surgery. ${ }^{1}$ Models of hepatic warm ischemia in rodents have been instrumental in elucidating mechanisms of injury during liver transplantation and hepatic ischemic/reperfusion injury.

In humans and primates, occlusion of the hepatoduodenal ligament (containing the portal vein, hepatic artery and the common bile duct) is well tolerated because of a more efficient portosystemic

Correspondence: Dr LL Moldawer, PhD, Department of Surgery, University of Florida College of Medicine, Room 6116, Shands Hospital, Box 100286, Gainesville, FL 32610-0286, USA.

E-mail: moldawer@surgery.ufl.edu

Presented at the 27th Annual Shock Society Meeting, Nova Scotia, Canada, 2004

Received 29 September 2004; revised and accepted 13 October 2004; published online 22 November 2004 collateral network through which splanchnic blood can return to the heart (Pringle maneuver ${ }^{2}$ ). Interruption of blood flow to the normothermic liver is safe for at least 60 min even without a venous bypass to decompress the vasculature proximal to the occlusion. ${ }^{3}$ In contrast, THI in rodents has been shown to produce several pathologic events, including splanchnic congestion, severe intestinal ischemia, mesenteric congestion and systemic shock. ${ }^{4}$ Consequently, these animals poorly tolerate even short periods of total hepatic ischemia. For example, $30 \mathrm{~min}$ of ischemia induces an immediate and delayed intestinal barrier failure, which results in significant increases in bacterial translocation and endotoxemia. This intestinal barrier failure is presumed to be due to portal stasis leading to intestinal congestion as well as temporary liver ischemia. Sixty minutes of hepatic ischemia without portal decompression in rats results in a high mortality with the animals displaying hyperamylasemia, congestive damage to the pancreas and portal 
endotoxemia due to increased small intestine permeability. ${ }^{5}$

In order to allow more in-depth study of the selective mechanisms of liver injury during warm hepatic ischemia in situ, investigators have developed rodent models of total hepatic ischemia that eliminate the confounding of splanchnic congestion and mesenteric hypertension. One model is the extracorporeal portosystemic shunt, ${ }^{6}$ which uses a polyethylene tube with $1 \mathrm{~mm}$ inner diameter. Although this model reduced intestinal congestion following total hepatic ischemia and reperfusion in rats, ${ }^{7}$ this technique is not feasible in mice due to their small size and vasculature. An alternative model is the subcutaneous ST method, ${ }^{8}$ which requires that 3 weeks prior to hepatic ischemia, the mouse spleen is placed into a subcutaneous pouch in order to induce portosystemic collateral blood vessel synthesis. Finally, some investigators have developed a model that creates a caudal shunt following a $30 \%$ hepatectomy, ${ }^{9}$ leaving a small remnant of the liver for the diverted blood flow. This creates a shunt, allowing sufficient blood to flow through the remnant while inducing ischemia to the remaining $70 \%$ of the liver.

The present study examined whether the two models were equivalent for use in studying murine total hepatic ischemia. We compared outcome, inflammatory response and hepatic injury with $30 \mathrm{~min}$ of total hepatic ischemia followed by 60 min of reperfusion in these two models.

\section{Materials and methods}

\section{Animals}

C57BL/6 mice 6-12-week-old females (Jackson Laboratories, Bar Harbor, ME, USA) were used for the study. Animals were housed for at least 6 days in a light- and temperature-controlled room to equilibrate to their surroundings prior to any procedures. Mice were provided access to rodent chow and water ad libitum. All surgical procedures were performed under anesthesia with the use of intraperitoneal injection of $35-50 \mathrm{mg} / \mathrm{kg}$ of sodium pentobarbital. An adequate plane of anesthesia was assumed when the animals were unable to right themselves after being placed on their backs, as well as when they were unable to respond to toe pinch. Procedures were carried out according to the University of Florida Institutional Animal Care and Use Committee guidelines. The laboratory adheres to the NIH Guiding Principles of Laboratory Animal Use and Care, as promulgated by the American Physiologic Society.

\section{Subcutaneous Splenic Transposition (ST)}

ST was performed as described by Bengmark et $a l^{8}$ in rats. Briefly, a $0.5 \mathrm{~cm}$ incision was made in the left subcostal arch. A subcostal pouch was created laterally in the subcutaneous tissue. Next, the spleen was pulled out and buried in the pouch. One stitch was used around the neck of the pouch.

Immediately after surgery and for the next 2 days, mice received a subcutaneous injection of $0.05-0.10 \mathrm{mg} / \mathrm{kg} /$ day of buprenorphine for analgesia. After 3 weeks, the portosystemic shunt developed through expansion of the epigastric vessels (Figure 1a, b). To assess the development of the collateral vessels, mice were killed immediately, and at $1,3,5,7,10,14$ and 21 days after transposition.

\section{Caudal Shunt with a $30 \%$ Hepatectomy (CS)}

Caudal shunt was performed as described by Yadav et al. ${ }^{9}$ Briefly, a midline incision was performed, extending from the xiphoid to the lower abdomen. The bowel loops were exteriorized and covered with saline-soaked gauze in order to prevent dehydration. Next, the left liver lobes, including the papillary lobe and the quadrate process, were isolated from their ligaments. The papillary and the quadrate process were ligated with 5-0 silk and resected. After the papillary lobe and the quadrate process resection, the caudate and the right lobes were isolated from their ligament, particularly the vena cava. The caudate lobe always surrounds the vena cava. Subsequently, these lobes were ligated and resected separately. According to the authors, the resected tissues represented less than $30 \%$ of the total hepatic mass.

\section{Measurement of Hepatic Inflow}

Injection of $0.2 \%$ 1,9-dimethylene blue (Sigma Aldrich Co., San Diego, LA, USA) via the superior mesenteric vein was used to evaluate the effect of ST on hepatic inflow during normal status (without Pringle maneuver) 14 days after ST. Under anesthesia, the superior mesenteric vein was cannulated with a 24-gauge catheter and perfused with blue dye (diluted at 1:500 ratio with normal saline) until sufficient staining of the perfused organs was obtained. After staining, mice were killed and their liver, intestine and spleens were harvested.

\section{Total Hepatic Ischemia and Reperfusion}

In the naive and the ST mice, total hepatic ischemia was accomplished by placing a microvascular clip (Miltex Inc., Bethpage, NY, USA) on the hepatoduodenal ligament. Total hepatic ischemia in the caudal shunt animals was created by applying microvascular clips to the respective vascular supply of the remaining lobes. After $30 \mathrm{~min}$ of total hepatic ischemia, the clamp was removed for a reperfusion time of $60 \mathrm{~min}$. 
92

a
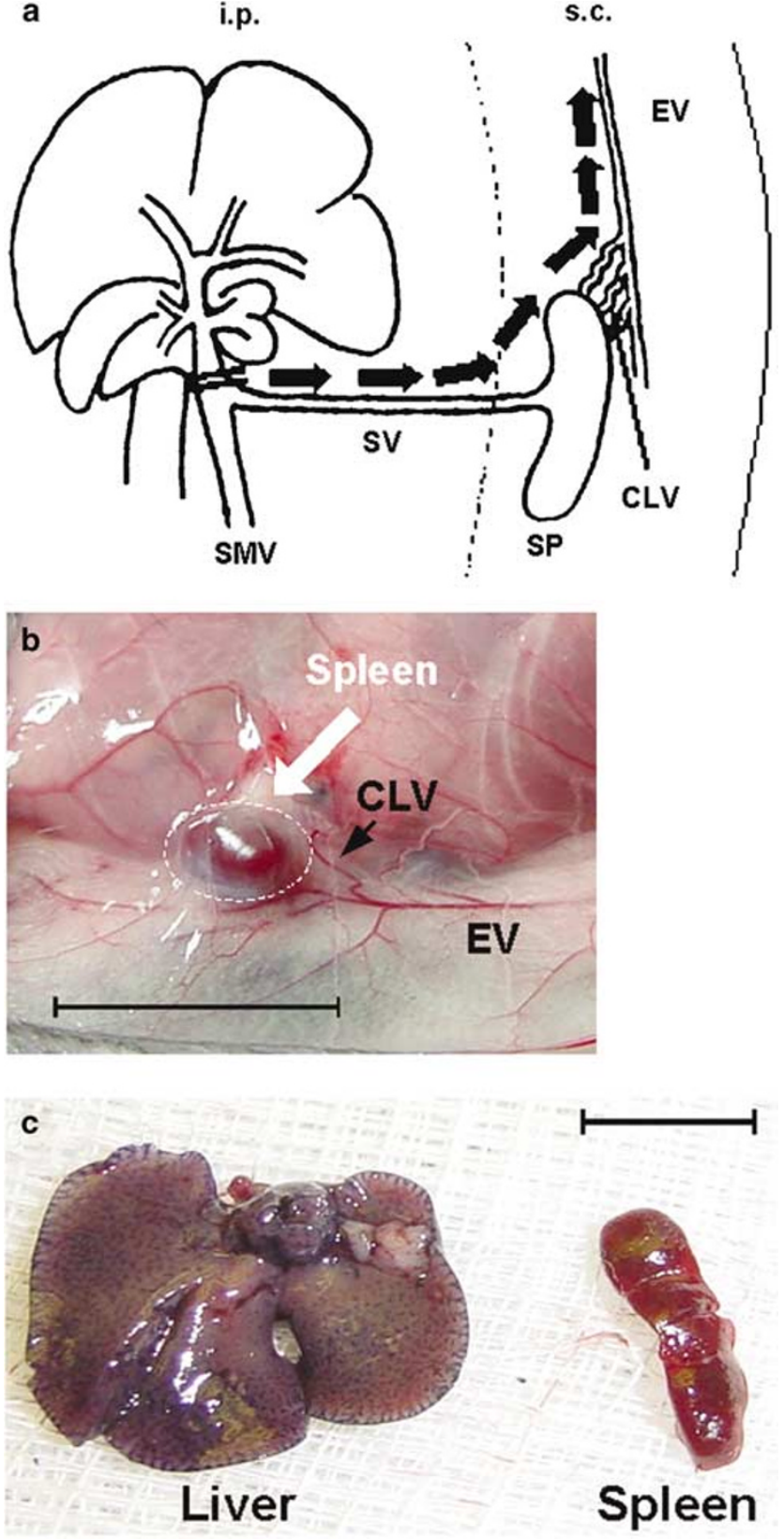

Figure 1 Subcutaneous splenic transposition. (a) Transposition of the spleen to the extraabdominal cavity. During total hepatic ischemia, portal blood drains to the epigastric vein via collateral vessels. The peritoneum is indicated as a dotted line. The solid line represents the skin. (b) Appearance of the collaterals. Increased capillary vessels are apparent around the spleen (indicated by black arrows). The white arrow points to the transposed spleen (dotted circled line). Scale bar, $1 \mathrm{~cm}$. (c) Injection of $0.2 \%$ 1,9-dimethylene blue via portal vein without Pringle maneuver (POD 21). Almost all the injected blue dye was trapped in the liver, and not in the spleen. This indicated that splenic transposition did not alter normal liver blood circulation. Scale bar, $1 \mathrm{~cm}$. SMV: superior mesenteric vein; SV: splenic vein; SP: spleen; EV: epigastric vein; CLV: collateral vessels.

\section{Functional Evaluation of Perisplenic Collaterals}

To confirm the effect of collateral circulation on portal hypertension, survival rates over a $24 \mathrm{~h}$ period were examined in mice by ligating the portal vein at various time points after ST $(2,5,7,14,21$ and 42 days, seven mice per group).

\section{Hepatic Ischemia and Experimental Groups}

Animals were randomly assigned to one of the following groups: (1) mice that underwent ST followed by a sham laparotomy for $90 \mathrm{~min}$ without total hepatic ischemia; (2) mice that underwent ST followed by total hepatic ischemia $(30 \mathrm{~min})$ and reperfusion (60 min); (3) mice that underwent a caudal shunt followed by sham laparotomy for $90 \mathrm{~min}$; (4) mice that underwent a caudal shunt followed by total hepatic ischemia $(30 \mathrm{~min})$ and reperfusion (60 min); (5) mice that underwent only a sham 90 min laparotomy and (6) mice that underwent total hepatic ischemia $(30 \mathrm{~min})$ and reperfusion (60 min) without any shunting (ST or caudal shunt). Immediately after total hepatic ischemia, all of the mice were administered $1 \mathrm{ml}$ of physiologic saline by dorsal subcutaneous injection. Mice were either observed for survival $(1 \mathrm{~h})$ or were killed to obtain blood, as well as liver tissue.

\section{Plasma Aspartate Aminotransferase (AST), Alanine Aminotransferase (ALT) and Interleukin-6 (IL-6)}

Whole blood $(0.5-1.0 \mathrm{ml})$ obtained by cardiac puncture was immediately centrifuged at $4000 \mathrm{rpm}$ for $10 \mathrm{~min}$. Serum AST/ALT was used as a marker of hepatocyte injury. Levels of enzyme activities were measured using the serum multiple biochemical analyzer, and were expressed as units/liter.

Serum IL-6 levels were determined by an enzymelinked immunoadsorbent assay (ELISA) using a mouse IL-6 MiniKit (KMIL6, Endogen Inc., Boston, MA, USA). ELISA for murine IL-6 was performed using flat bottom high-binding polystyrene microtiter plates (Corning Incorporated, Corning, NY, USA). Strepavidan HRP (RPN1231V Amersham Inc., Rockford, IL, USA) was used at 1:1000 dilutions. TMB substrate (T8665 Sigma Fine Chemicals, St Louis, MO, USA) was used for detection of cytokines.

\section{Histology}

For histologic examination, tissue samples from the hepatic left lateral lobe were obtained. The samples were fixed in $10 \%$ neutral-buffered formalin, embedded in paraffin, sectioned at $5 \mu \mathrm{m}$ and stained with hematoxylin and eosin for evaluation of necrosis and apoptosis. The number of apoptotic hepatocytes and sinusoidal endothelial cells were counted in 10 high-power fields $(\times 400)$ from pericentral regions only. Apoptotic and necrotic hepatocytes were determined on hematoxylin- and eosin-stained slides as previously described. ${ }^{10-13}$ Apoptotic cells were identified in hematoxylinand eosin-stained specimen by morphological criteria such as cell shrinkage, chromatin condensation 
with margination around the nuclei and the formation of apoptotic bodies (Figure 6). Necrotic cells were identified by cell disruption, increased cell volume, loss of architecture and vacuolization (Figure 6).

Apoptosis and necrosis were estimated by counting the number of apoptotic or necrotic cells per fixed number of high-magnification microscopic fields, in a blinded fashion.

Sixty Minutes Survival after Total Hepatic Ischemia of Two Durations in ST and Caudal Shunt

To determine survival rate, the abdominal incision was closed after completion of either 30 or $60 \mathrm{~min}$ of total hepatic ischemia, respectively, and the number of mice surviving for $60 \mathrm{~min}$ was recorded for both groups.

\section{Twenty Four Hours Survival after 30 min of Total Hepatic Ischemia and Reperfusion in Naive, ST and Caudal Shunt Mice}

To determine survival rate, the abdominal incision was closed after completion of total hepatic ischemia and the number of mice surviving for $24 \mathrm{~h}$ was recorded for all groups ( $n=5$ per group).

\section{Statistics}

Data are presented as the mean \pm standard error of the mean. Comparisons among multiple groups were performed with one-way ANOVA followed by Bonferroni post hoc tests. In the case where a nonparametric test was required, data were analyzed with the Kruskal-Wallis ANOVA followed by Dunn's Multiple Comparisons Test. Survival proportions were analyzed by $\chi^{2}$ analysis or Fisher Exact test. Differences with a $P$ less than 0.05 were considered significant.

\section{Results}

\section{Splenic Transposition}

In general, the procedure required less than $3 \mathrm{~min}$ to perform and almost all cases took less than $1 \mathrm{~min}$. The surgical procedure was not associated with any bleeding or subcutaneous infection. Subcutaneous collaterals were detected macroscopically by the 14th postoperative day (Figure 1b).

Complications were observed in two of 30 ST animals $(6.7 \%)$. In both cases, the spleen retracted into the abdominal cavity. In the 30 cases performed, there was no mortality due to the procedure or during the subsequent 21-day collateralization period.

Infusion of $0.2 \%$ 1,9-dimethylene blue via the portal vein was detected in the whole liver im- mediately, and not in the spleen. This phenomenon appeared even if the infusion pressure was increased (Figure 1c). This absence of splenic staining is consistent with the hepatic circulation not being significantly altered by the ST procedure.

For the preliminary evaluation of functional perisplenic collaterals, the portal vein was ligated. Figure 2 illustrates the 24 -h survival rate in reference to the time period between ST and surgical ligation of the portal vein. For example, all mice died when portal vein ligation for $30 \mathrm{~min}$ was performed only 2 days after ST. In contrast, $14 \%$ of the mice survived when portal vein ligation was performed 5 days after ST, 29\% survived 6 days and $71 \%$ survived when the portal vein was ligated 14 days after ST. All mice undergoing portal vein ligation survived when it was performed 21 and 42 days after ST $(P<0.001$ vs 2 days after ST). Thus, these studies confirm that at least 3 weeks are required for appropriate collaterals to form allowing the ST model to work effectively. ${ }^{14}$

\section{Caudal Shunting}

The caudal shunting model takes approximately 10-30 min to perform. The complication rate was $30 \%$ (six of 20 cases), and included three cases of bleeding from the portal vein, vena cava or liver, as well as disturbances of the liver and/or vena cava circulation.

\section{Sixty Minutes Survival Rate after Total Hepatic Ischemia}

Mice that underwent CS followed by $30 \mathrm{~min}$ of total hepatic ischemia and 60 min of reperfusion had a significantly poorer survival rate than mice that underwent ST followed by total hepatic ischemia

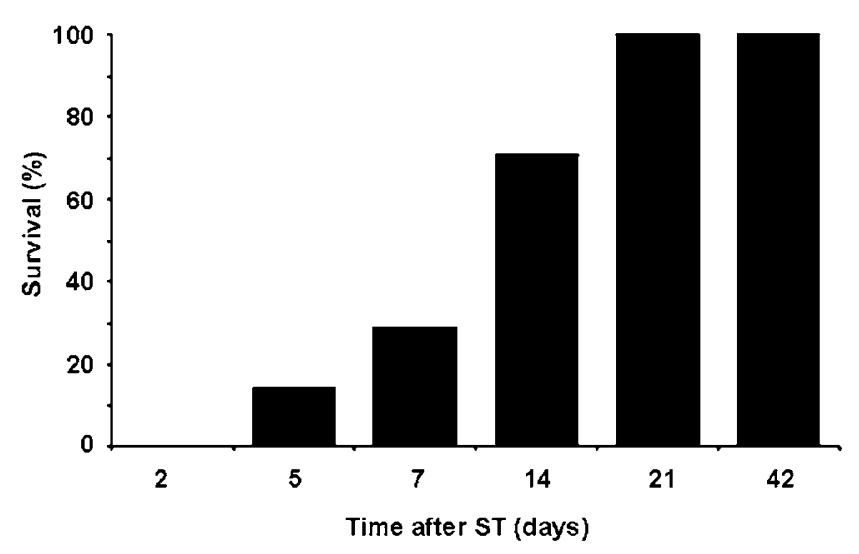

Figure 2 Twenty four hours survival following portal vein ligation applied at various time intervals after splenic transposition $(2,5,7,14,21$, and 42 days, seven mice per group). All mice undergoing portal vein ligation survived when it was performed 21 and 42 days after splenic transposition $(P<0.001$ compared with 2 days after splenic transposition, Fisher Exact Test). 


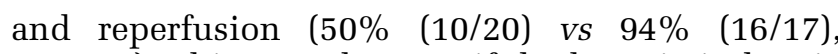
$P<0.01)$. This was also true if the hepatic ischemia time was extended to $60 \mathrm{~min}(28 \%(2 / 7)$ survival vs $92 \%$ (12/13), $P<0.01)$.

\section{Twenty Four Hours Survival after 30 min of Total Hepatic Ischemia and Reperfusion in Naive Mice, ST and CS}

In naive mice, the $24 \mathrm{~h}$ survival rate after $30 \mathrm{~min}$ of total hepatic ischemia and reperfusion was $20 \%$ $(1 / 5)$. In ST and CS mice, the $24 \mathrm{~h}$ survival rate after 30 min of total hepatic ischemia and reperfusion was $100 \%(5 / 5)$ and $20 \%(1 / 5)$, respectively. There was a significant difference in survival among the three groups ( $\chi^{2}$ test, $P<0.05$ ) (Figure 3 ).

\section{Serum ALT Levels}

Serum AST/ALT concentrations in control animals and animals that underwent splenic transposition alone did not significantly differ (Figure 4a,b). However, the mice that underwent the caudal shunting alone demonstrated significantly elevated AST/ALT concentrations when compared with either ST or control mice that did not undergo hepatic ischemia $(P<0.05)$ (Figure $4 \mathrm{a}, \mathrm{b})$, indicating some hepatocellular injury associated with the caudal shunting procedure alone. In all three groups of mice, (controls, ST and CS), however, $30 \mathrm{~min}$ of total hepatic ischemia followed by $60 \mathrm{~min}$ of reperfusion injury significantly increased the AST/ ALT concentrations. Moreover, the levels of AST/ ALT in mice that underwent CS with total hepatic ischemia and reperfusion were significantly higher than similar mice with total hepatic ischemia and

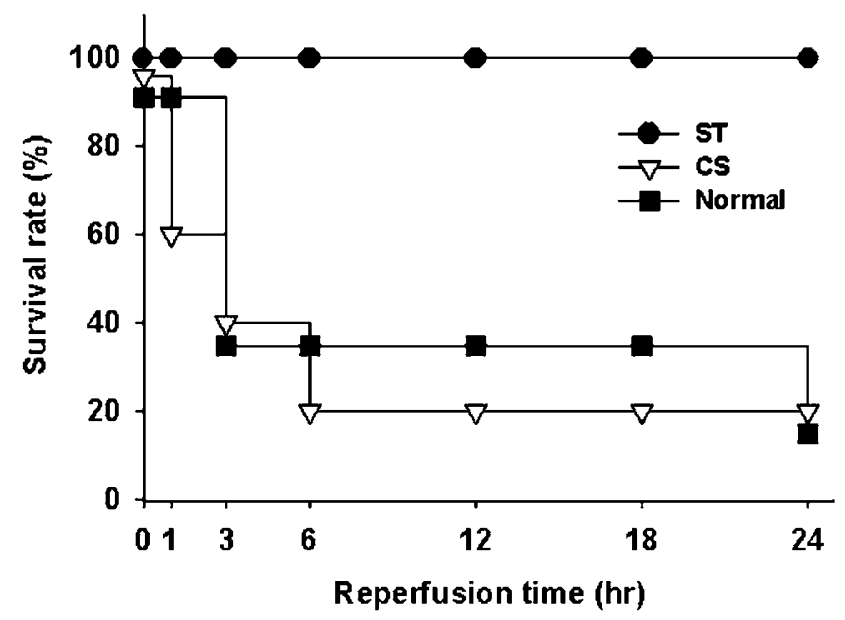

Figure 3 Twenty four hours survival after $30 \mathrm{~min}$ of THI and reperfusion in the three groups. In naive mice, the $24 \mathrm{~h}$ survival rate after $30 \mathrm{~min}$ of THI and reperfusion was $20 \%(1 / 5)$. In ST and CS mice, the $24 \mathrm{~h}$ survival rates after $30 \mathrm{~min}$ of THI and reperfusion were $100 \%(5 / 5)$ and $20 \%(1 / 5)$, respectively. There was significant difference in $24 \mathrm{~h}$ survival among the three groups $\left(P<0.05\right.$, by $\chi^{2}$ test). ST: splenic transposed mice; CS: caudal shunting mice.

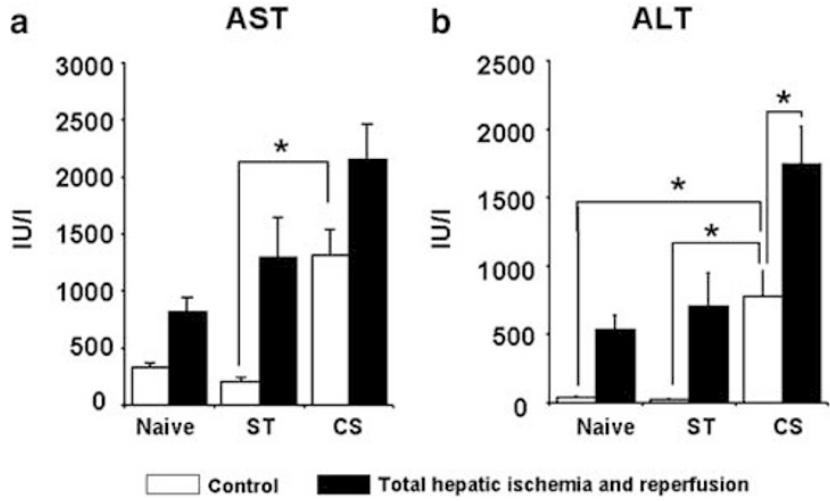

Figure 4 Serum AST/ALT levels. Serum levels of ALT were significantly higher in THI and reperfusion with caudal shunt $\left({ }^{*} P<0.05\right.$ caudal shunt without THI and reperfusion vs caudal shunt with THI and reperfusion). Caudal shunt also increased baseline injury in non-THI animals $\left({ }^{*} P<0.05\right.$, AST/ALT: ST without THI and reperfusion vs caudal shunt without THI and reperfusion, ALT: naive animals without THI and reperfusion vs caudal shunt without THI and reperfusion). ST: splenic transposed mice; CS: caudal shunting mice; THI: total hepatic ischemia.

reperfusion that had undergone no prior procedure or the splenic transposition procedure $(P<0.05)$ (Figure 4a,b). This latter finding suggests that the increased AST/ALT concentrations with caudal shunting followed by total hepatic ischemia and reperfusion injury may reflect an additive injurious response to the two procedures.

\section{Serum Levels of IL-6 (ELISA)}

The serum lL-6 levels in both naive mice and splenic transposed mice without total hepatic ischemia were not detectable. On the other hand, the mice undergoing caudal shunting without total hepatic ischemia exhibited slightly increased serum IL-6 concentrations (Figure 5), albeit levels did not reach statistical significance.

The serum IL-6 levels 60 min after reperfusion in naive mice and CS mice that underwent total hepatic ischemia and reperfusion were significantly increased compared with those that did not receive total hepatic ischemia $(P<0.05)$ (Figure 5). Although the serum IL-6 levels in ST mice with total hepatic ischemia followed by reperfusion were similarly increased, the increases did not reach statistical significance.

\section{Apoptosis}

In naive mice, apoptotic and necrotic hepatocytes were only rarely observed. The sinusoids appeared normal and there was no congestion. There were only $0.4 \pm 0.1 \%$ apoptotic hepatocytes in 10 highpowered fields (Figures 6c and 7a). In mice undergoing ST without total hepatic ischemia, the rate of apoptosis was similar to control animals with $0.5 \pm 0.1 \%$ of total hepatocytes in 10 high-powered 


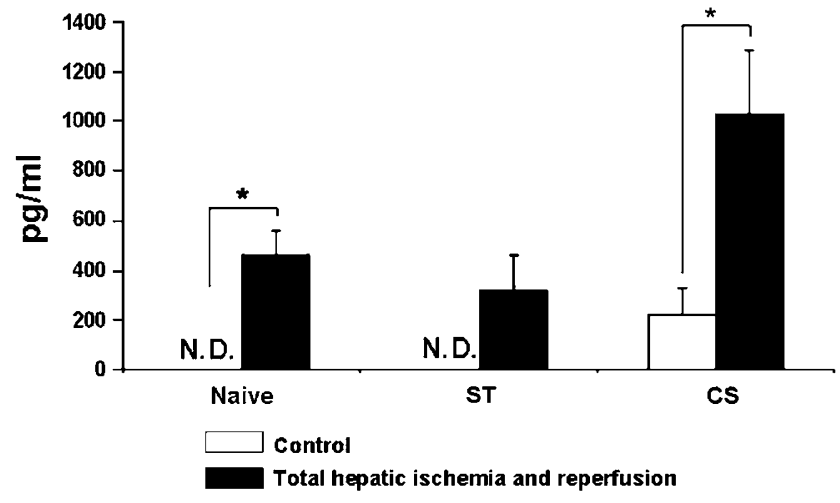

Figure 5 Serum IL-6 levels. After THI and 60 min of reperfusion, the serum IL-6 levels in naive mice and caudal shunting mice were significantly increased compared with those that did not receive total hepatic ischemia $\left({ }^{*} P<0.05\right.$, Kruskal-Wallis). N.D. $=$ not detected

fields (Figure 7a). In contrast, after total hepatic ischemia/reperfusion in mice with splenic transposition, the rate of apoptosis significantly increased to $1.4 \pm 0.2 \%$ of total hepatocytes in 10 highpowered fields $(P<0.001$ Bonferroni tests) (Figures $6 d$ and 7a).

In mice undergoing caudal shunting without total hepatic ischemia, the rate of apoptosis was $0.8 \pm 0.2 \%$ of total hepatocytes in 10 high-powered fields (Figure 7a). This was modestly, but insignificantly, increased, when compared to controls. After total hepatic ischemia and reperfusion in mice with CS, apoptotic hepatocytes constituted $2.5 \pm 0.2 \%$ of total hepatocytes in 10 high-powered fields $(P<0.001)$ (Figures 6e and 7a). These numbers of apoptotic cells in mice following CS after total hepatic ischemia/reperfusion were significantly higher compared with those of splenic transposition mice $(P<0.001)$ (Figure 7a). The apoptotic hepatocytes were located randomly within the hepatic lobules and in no case were found as clusters of several cells.

\section{Necrosis}

The appearance of a necrotic hepatocyte in ST mice without total hepatic ischemia was rare, with $0.3 \pm 0.1 \%$ of total hepatocytes in 10 high-powered fields (Figure 7b). Rates were similar to those seen in healthy controls. After total hepatic ischemia/reperfusion in mice with splenic transposition, the rate of necrosis significantly increased to $17.8 \pm 1.3 \%$ of total hepatocytes in 10 high-powered fields $(P<0.05)$ (Figures $6 \mathrm{~d}$ and $7 \mathrm{~b})$.

In mice with CS but without total hepatic ischemia, the rate of necrosis was modestly, but insignificantly, increased with $2.5 \pm 0.3 \%$ of total hepatocytes in 10 high-powered fields (Figure 7b). After total hepatic ischemia/reperfusion in mice with CS, necrotic hepatocytes constituted
$17.1 \pm 0.9 \%$ of total hepatocytes in 10 high-powered fields. There were significant differences between both nonischemic and total hepatic ischemia/reperfusion groups, regardless of whether the animals had undergone ST or CS $(P<0.05)$ (Figures 6e and $7 b)$. However, no difference in the rate of necrosis was seen in ST vs CS mice undergoing total hepatic ischemia and reperfusion.

\section{Discussion}

The purpose of this investigation was to evaluate experimental models of total hepatic ischemia and reperfusion in mouse. Rodent total hepatic ischemia is associated with splanchnic congestion, severe intestinal ischemia and mesenteric congestion, all of which can lead to systemic shock and death. Thus, alternative methods are required to induce purposeful total hepatic ischemia in rodents without the confounding variables of intestinal congestion. ST and CS were evaluated as a means to provide a simple and safe method of total hepatic ischemia in mouse.

The functional concept of decreasing portal hypertension by transposing the spleen to the subcutis to develop a collateral vasculature was initially described by Bengmark et al. ${ }^{8}$ Although this principle of portosystemic shunt has been associated with increased frequency of encephalopathy in clinical practice, ${ }^{15}$ similar complications in rodents were only observed when the portal vein was ligated. ${ }^{16}$

To assess the efficiency of splenic transposition, we performed a preliminary time-course response in 20 mice. Both an anatomic exploration of new vessel growth and a functional response to total hepatic ischemia and reperfusion injury were determined. Consistent with previous reports, spindle-shaped subcutaneous collateral vessels were visualized from around the spleen to the epigastric vessels after 2 weeks post-transposition. An increase of the time interval between ST and ligation of the portal vein did not reveal any further gain in development of potential parasplenic collaterals. ${ }^{8}$

Initially, we examined the 24 -h survival rate of mice undergoing acute portal obstruction after various time-intervals following ST. Our hypothesis was that mortality would be secondary to intestinal congestion and injury (Figure 2). All mice survived ligation of the portal vein when it was performed 21 and 42 days after ST. Earlier periods were associated with a time-dependent increase of mortality. Based on these preliminary data, we decided to examine total hepatic ischemia in mice at least 21 days after ST.

To assess potential deviations in hepatic inflow caused by the portosystemic shunt, we administered $0.2 \%$ 1,9-dimethylene blue via the superior mesenteric vein, to examine whether there were gross changes in hepatic circulation. Infusion of blue dye 
immediately stained the entire liver. In addition, there was no staining of the spleen (comparable to a naive mouse), even if the pressure of infusion was raised (Figure 1c). Moreover, splenomegaly and intra-abdominal collaterals (eg splenorenal shunt, paraesophageal veins and esophageal varices) were never observed in this model. Portal hypertension has been shown to associate with hepatic dysfunc-
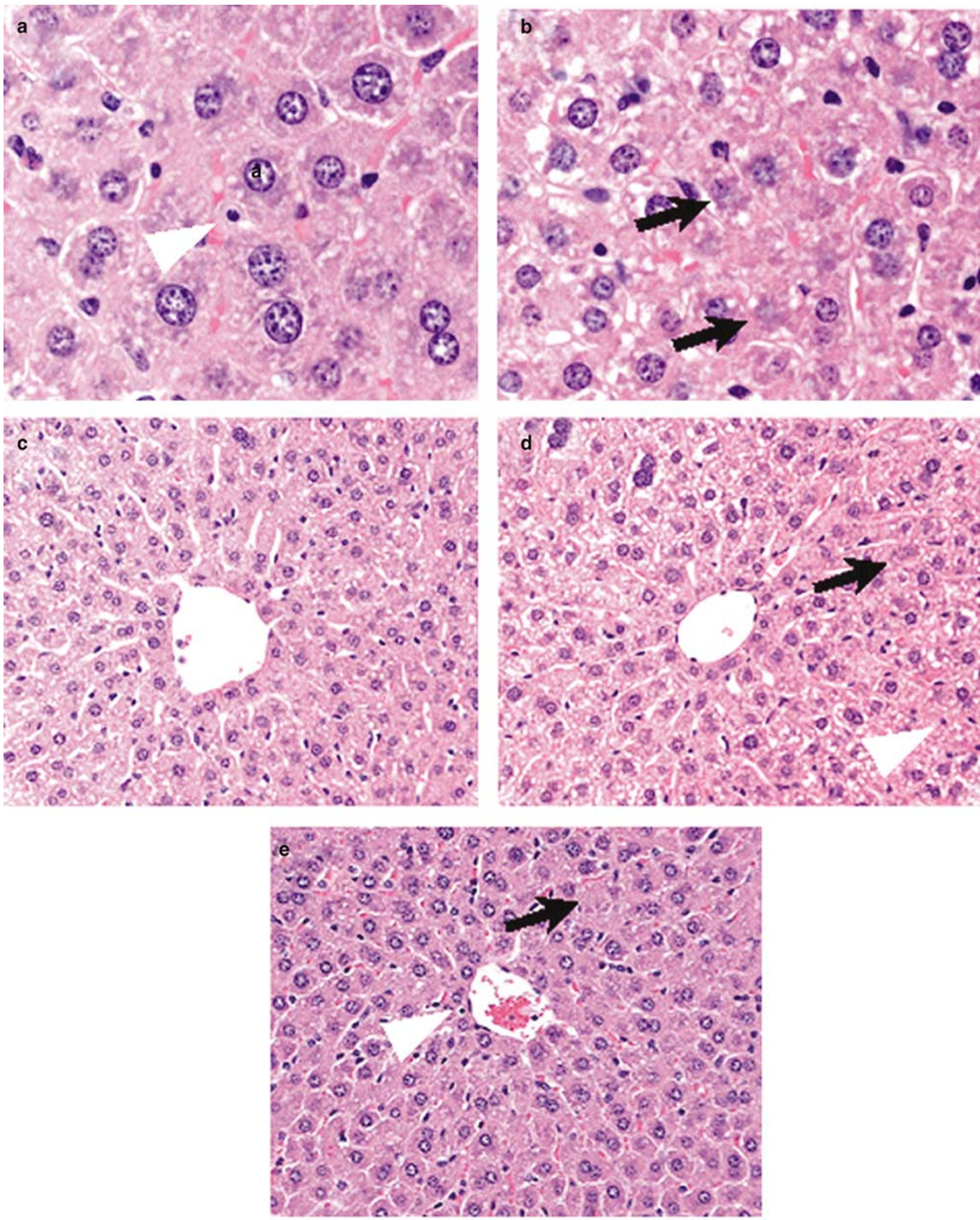


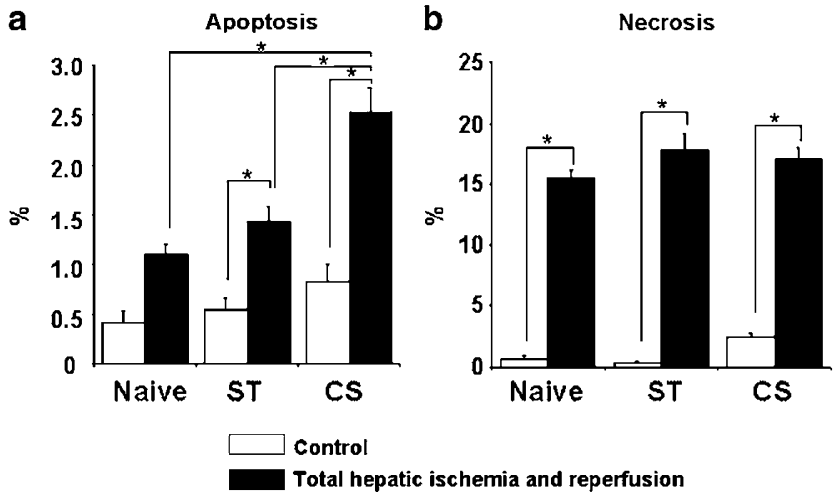

Figure 7 Hepatocyte apoptosis/necrosis after total hepatic ischemia and 60 min of reperfusion. (a) Levels of hepatocyte apoptosis. Although CS with THI and reperfusion induce significantly more apoptosis compared with naive mice with THI and reperfusion, splenic transposed mice with THI and reperfusion and CS without THI and reperfusion. These levels may not be biologically relevant or allow detection by caspase assay $\left({ }^{*} P<0.001\right.$ Bonferroni tests). (b) Levels of hepatocyte necrosis. All models significantly increase necrosis of the liver with THI and reperfusion $\left({ }^{*} P<0.001\right.$ Bonferroni tests). ST: splenic transposition; CS: caudal shunting.

tion, ${ }^{15,17,18}$ but no abnormal levels of serum AST/ ALT in mice were observed following ST.

CS is an alternative approach to generate a portosystemic shunt. Despite other reports stating this to be a simple and rapid procedure, performance of CS holds a rather complex surgical technique, due to the topography of the caudate lobe surrounding the vena cava. As shown in Figures 4 and 5, the actual surgical procedure produced some modest hepatocellular injury, which was not seen with the splenic transposition. Furthermore, this injury and inflammation appeared to be additive when superimposed on a subsequent total hepatic ischemia and reperfusion injury.

The results of this study demonstrate that both methods can be used to explore ischemia and reperfusion liver injury in mouse. The appearance of AST/ALT after hepatic ischemia/reperfusion injury in both groups (ST and CS) was consistent with hepatic injury. Not surprisingly, ST alone did not cause liver injury.

It has been reported that apoptosis plays an important role in hepatic ischemia and reperfusion injury. ${ }^{10,12}$ In the present study, we used morphological criteria from hematoxylin- and eosin-stained preparations as the gold standard for the identification of apoptotic cells. ${ }^{10-13}$ We initially employed TUNEL staining (data not shown), but subsequently abandoned it because of its lack of sensitivity and inability to discriminate among the low levels of apoptosis seen in the present study. In our study, apoptotic hepatocytes were detected only rarely in $0.4-0.5 \%$ of naive and splenic transposed mice without total hepatic ischemia, respectively. In CS, the percentage of apoptotic cells tended to be higher than in splenic transposed mice, but was still generally low. Consistent with modest but increased levels of AST/ALT, CS mice without total hepatic ischemia showed a higher rate of necrosis than control or ST mice. This suggests that the CS itself may cause preischemic hepatic injury through hepatocellular necrosis. Interestingly, in naive mice, the rate of apoptosis and necrosis following total hepatic ischemia was similar to ST, which did not appear to correlate with the higher mortality observed in the naive mouse group (compare Figures 3 and 7). A possible explanation for this might be found in the intestinal reaction rather than the assumed hepatic injury, as total hepatic ischemia without portosystemic shunt resulted in significant portal hypertension and intestinal congestion. This physiological derangement enables immediate breaching of pathogenic vectors (eg bacteria) across the intestinal barrier with the subsequent risk of higher mortality. ${ }^{4}$ As shown in Figure $3,80 \%$ of naive mice that underwent $30 \mathrm{~min}$ of total hepatic ischemia without any method of portosystemic shunting died within $24 \mathrm{~h}$ as a result of this procedure.

Thus, we have demonstrated that splenic transposition appears to be a superior model to the caudal shunt for studying total hepatic ischemia in mice. ST is a simple, safe and suitable model for studying total hepatic ischemia without confounding due to liver manipulation, and is not associated with significant mortality. ST also results in decreased hepatocellular injury when compared to CS, and thus offers pathophysiological advantages for reliable assessments of total hepatic ischemic injury.

Figure 6 Hematoxylin- and eosin-stained livers from mice subjected to total hepatic ischemia and reperfusion. (a) The white arrowhead points to an apoptotic hepatocyte. Magnification is $\times 1000$. (b) The black arrow points to a necrotic hepatocyte. Magnification is $\times 1000$. (c-e) representative pericentral regions (magnification $\times 400$ ) from naive, splenic transposition and caudal shunt mice. Calculations based on 10 high-powered fields from pericentral regions only. The white arrowhead indicates apoptotic hepatocyte. The block arrow indicates necrotic hepatocyte. (c) Naive mouse liver (pericentral region): photomicrograph of a representative liver specimen from a naive mouse without total hepatic ischemia. The numbers of apoptotic hepatocytes constituted $0.4 \pm 0.1 \%$ of total hepatocytes in 10 high-powered fields. Necrotic hepatocytes constituted $0.3 \pm 0.1 \%$ of total hepatocytes. (d) Splenic transposition with THI and reperfusion (pericentral region): photomicrograph of a representative liver specimen from a mouse undergoing splenic transposition 21 days earlier, followed by $30 \mathrm{~min}$ of total hepatic ischemia and $60 \mathrm{~min}$ of reperfusion. The numbers of apoptotic hepatocytes constituted $1.4 \pm 0.1 \%$ of total hepatocytes. Necrotic hepatocytes constituted $17.8 \pm 1.3 \%$ of total hepatocytes. (e) CS, with THI and reperfusion (pericentral region): photomicrograph of a representative liver specimen from a mouse undergoing CS, followed immediately by $30 \mathrm{~min}$ of total hepatic ischemia and $60 \mathrm{~min}$ of reperfusion. Apoptotic hepatocytes constituted $2.5 \pm 0.2 \%$ of total hepatocytes. Necrotic hepatocytes constituted $17.1 \pm 0.9 \%$ of total hepatocytes. 


\section{Acknowledgements}

The authors gratefully acknowledge the support of Grants R37 GM-40561-15, and R01 GM-63041-03, awarded by the National Institute of General Medical Sciences, U.S.P.H.S. PAE was supported by a NIGMS-funded T32 training grant, awarded by the National Institute of General Medical Sciences.

\section{References}

1 Hasselgren PO. Prevention and treatment of ischemia of the liver. Surg Gynecol Obstet 1987;164:187-196.

2 Pringle J. Notes on the arrest of hepatic hemorrhage due to trauma. Ann Surg 1908;48:541-549.

3 Huguet C, Gavelli A, Chieco PA, et al. Liver ischemia for hepatic resection: where is the limit? Surgery 1992;111:251-259.

4 Yamauchi H, Baca I, Mittmann U, et al. Postischemic liver damage in rats: effect of some therapeutic interventions on survival rate. Tohoku J Exp Med 1982;138:63-70.

5 Ochiai H, Nakamura S, Suzuki S, et al. Pancreatic damage resulting from temporary portal triad interruption during partial hepatectomy: protective effect of a prostaglandin I2 analogue. J Surg Res 1997;73:129-136.

6 Hirasawa H, Chaundry IH, Baue AE. Improved hepatic function and survival with adenosine triphosphatemagnesium chloride after hepatic ischemia. Surgery 1978;83:655-662.

7 Suzuki S, Nakamura S, Sakaguchi T, et al. Pathophysiological appraisal of a rat model of total hepatic ischemia with an extracorporeal portosystemic shunt. J Surg Res 1998;80:22-27.
8 Bengmark S, Borjesson BT, Olin T. Development of portasystemic shunts after subcutaneous transposition of the spleen in the rat. Am J Surg 1973;125:757-762.

9 Yadav SS, Gao W, Harland RC, et al. A new and simple technique of total hepatic ischemia in the mouse. Transplantation 1998;65:1433-1436.

10 Jaeschke H, Lemasters JJ. Apoptosis versus oncotic necrosis in hepatic ischemia/reperfusion injury. Gastroenterology 2003;125:1246-1257.

11 Jolly PD, Smith PR, Heath DA, et al. Morphological evidence of apoptosis and the prevalence of apoptotic versus mitotic cells in the membrana granulosa of ovarian follicles during spontaneous and induced atresia in ewes. Biol Reprod 1997;56:837-846.

12 Gujral JS, Bucci TJ, Farhood A, et al. Mechanism of cell death during warm hepatic ischemia-reperfusion in rats: apoptosis or necrosis? Hepatology 2001;33: 397-405.

13 Kerr JF, Wyllie AH, Currie AR. Apoptosis: a basic biological phenomenon with wide-ranging implications in tissue kinetics. Br J Cancer 1972;26:239-257.

14 Jaffe V, Sandin B, Wilkins RA. Radiological definition of a new model of portal diversion in the rat. Br J Radiol 1990;63:615-619.

15 Williams R. Portal hypertension in idiopathic tropical splenomegaly. Lancet 1966;1:329-333.

16 Maddison JE, Dodd PR, Morrison M, et al. Plasma GABA, GABA-like activity and the brain GABAbenzodiazepine receptor complex in rats with chronic hepatic encephalopathy. Hepatology 1987;7:621-628.

17 Okudaira M, Ohbu M, Okuda K. Idiopathic portal hypertension and its pathology. Semin Liver Dis 2002;22:59-72.

18 Bunch SE, Johnson SE, Cullen JM. Idiopathic noncirrhotic portal hypertension in dogs: 33 cases (19821998). J Am Vet Med Assoc 2001;218:392-399. 